\section{(2) OPEN ACCESS}

\title{
Visual acuity outcomes and anti-VEGF therapy intensity in macular oedema due to retinal vein occlusion: a real-world analysis of 15613 patient eyes
}

\author{
Thomas Ciulla (1) , ${ }^{1,2}$ John S Pollack, ${ }^{3}$ David F Williams ${ }^{4}$
}

\begin{abstract}
${ }^{1}$ Retina Service, Midwest Eye Institute, Indianapolis, Indiana, USA

${ }^{2}$ Indiana University School of Medicine, Indianapolis, Indiana, USA

${ }^{3}$ Rush University, Chicago, Illinois, USA

${ }^{4}$ University of Minnesota, Minneapolis, Minnesota, USA
\end{abstract}

\section{Correspondence to}

Thomas Ciulla, 10300 North Illinois Street, Indianapolis, IN 46290, USA;

thomasciulla@gmail.com

Received 29 June 2020

Revised 25 August 2020

Accepted 13 September 2020
Check for updates

(C) Author(s) (or their employer(s)) 2020. Re-use permitted under CC BY-NC. No commercial re-use. See rights and permissions. Published by BMJ.

To cite: Ciulla T, Pollack JS, Williams DF. Br J

Ophthalmol Epub ahead of print: [please include Day

Month Year]. doi:10.1136/ bjophthalmol-2020-

317337
ABSTRACT

Background/Aims To assess visual acuity (VA) outcomes and antivascular endothelial growth factor (anti-VEGF) therapy intensity in retinal vein occlusion (RVO)-related macular oedema (ME).

Methods A retrospective study was completed in treatment-naïve patients with RVO-related ME from 2013 to 2019, using the Vestrum Health Retina Database. Results Mean baseline age was 72.4 years and $54 \%$ were women. In 6 months, in 8876 eyes with branch retinal vein occlusion (BRVO)-related ME, after a mean of 4.5 anti-VEGF injections, VA increased by 9.4 letters ( $95 \%$ confidence interval (Cl) for change in $V A+8.94$ to $+9.78, p<0.001$ ) from a baseline of 55.1 letters. In 6737 eyes with central retinal vein occlusion (CRVO)-related $M E$, after a mean of 4.6 anti-VEGF injections over 6 months, VA improved by 9.2 letters $(95 \% \mathrm{Cl}+8.50$ to $+9.87, \mathrm{p}<0.001)$ from a baseline of 37.2 letters. In 1 year, VA gain was similar (BRVO: 7.4 injections, +8.1 letters, $95 \% \mathrm{Cl}+7.55$ to $+8.57, \mathrm{p}<0.001$; CRV0: 7.6 injections, +7.1 letters, $95 \% \mathrm{Cl}+6.31$ to +7.95 , $\mathrm{p}<0.001)$. In 6 months and 1 year, mean letters gain increased with number of anti-VEGF injections. Patient eyes with baseline VA of 20/40 or better tended to lose VA in 1 year.

Conclusion Mean change in VA correlates with treatment intensity, but patients with better VA at presentation are susceptible to vision loss, reflecting a ceiling effect. Assessed with the same database, VA gains compare favourably with 1-year VA gains in neovascular age-related macular degeneration and diabetic ME, but exhibit a larger gap when compared with corresponding randomised controlled trials.

Macular oedema (ME) due to retinal vein occlusion (RVO) represents a common cause of vision loss globally. According to the 2017 American Society of Retina Specialists Preference and Trends survey, over $90 \%$ of US and ex-US retina physicians choose antivascular endothelial growth factor (anti-VEGF) agents as first-line therapy for RVO-related ME with moderate vision loss. ${ }^{1}$ In randomised clinical trials (RCTs), three anti-VEGF agents, aflibercept (Eylea; Regeneron), ranibizumab (Lucentis; Genentech) and off-label bevacizumab (Avastin; Genentech), have yielded meaningful improvement of vision in this disorder.

As healthcare systems increasingly focus on value, real-world outcomes progressively assume greater importance, specifically to benchmark therapies and healthcare providers for quality and reimbursement. Nonetheless, there have been few large clinical outcomes studies of anti-VEGF therapy for RVO-related ME.

In the present study, 6-month and 1-year outcomes were evaluated using a large database of aggregated, longitudinal de-identified electronic medical records (EMRs) from a demographically and geographically diverse panel of US retina specialists (Vestrum Health Retina Database, Naperville, Illinois, USA). Treatment-naïve patients with RVO-related ME who received anti-VEGF therapy between 2013 and 2019 were included in this study. These outcomes in RVO-related ME were also compared with those previously described in neovascular age-related macular degeneration (nAMD) and diabetic macular oedema (DME), using the same database and similar analysis methodology. ${ }^{2-5}$

\section{METHODS}

\section{Database}

The database has been described in prior publications. $^{2-5}$ The Vestrum Health Retina Database holds de-identified visit data from approximately 1.5 million unique patients with over 11 million encounters. The panel of 350 US retina specialists are diversified geographically in the Southeast (24\%), Mid-Atlantic (24\%), West (20\%), Southwest (12\%), Northeast (8\%), Great Lakes (7\%) and North Central (4\%) regions. These practices are based in urban (65\%), suburban (32\%) and rural (3\%) settings. Aggregated data includes inoffice and outpatient medications, exam findings including visual acuity (VA) and intraocular pressure, diagnostic test interpretations, systemic and ophthalmic diagnoses, medical and surgical treatments, and adverse events. A proprietary deidentification process substitutes alphanumeric codes for patient identifiers. The system updates the database weekly. A previously described ETDRS approximation formula, $85+50 \times \log$ (Snellen fraction), derives VA score. ${ }^{6}$

\section{Study design and dates and inclusion and exclusion criteria}

This analysis retrospectively assessed 6-month and 1-year VA outcomes and associations with antiVEGF therapy intensity in treatment-naïve US patients with RVO-related ME between 2013 and 2019. Indiana University institutional review board exempted this study from review because it involved only the assessment of pre-existing de-identified 
data. This analysis involved treatment-naïve patients with RVOrelated ME who underwent anti-VEGF treatment between January 2013 and October 2019, if they received at least one antiVEGF injection and had follow-up data through 6 months and 1 year for each of these respective cohorts. This analysis excluded patients with other retinal diagnoses. Age, gender, VA and antiVEGF agents were extracted from the database.

\section{Analysis}

Analysis methods were similar to previous studies, which facilitated comparison of anti-VEGF treatment outcomes in RVOrelated ME with those previously described in other disease states using the same database. ${ }^{2-5}$ Outcomes were analysed at the patient eye level, separately for branch retinal vein occlusion (BRVO)related $\mathrm{ME}$ and central retinal vein occlusion (CRVO)-related ME. As a sensitivity analysis, main outcome measures were assessed, excluding patients with RVO-related ME who underwent bilateral anti-VEGF treatment during the study period. The study analysed baseline characteristics with descriptive statistics, including mean values for baseline age and VA (letters). These baseline characteristics were also stratified by initial anti-VEGF agents.

The study assessed, as main outcome measures, mean 6-month and 1-year VA change from baseline with 95\% CIs and nominal pvalues, using paired t-tests. For all eligible patients, mean 6-month and 1-year VA change was determined and stratified by initial anti-VEGF agents. As a sensitivity analysis, mean 6-month and 1-year VA change was also determined after excluding those patients who switched from initial therapy.

The study assessed mean and median 6-month and 1-year injection frequency. Distribution of injection frequency over these time periods was assessed and charted on a histogram, between 1 and 7 for the 6-month time period and between 1 and 13 for the 1-year time period. The study assessed for baseline age and VA relationships with 6-month and 1-year injection frequency, through stratification by injection frequency. Stratification separately by injection frequency and by baseline VA was carried out to assess resulting mean 6-month and 1-year VA change from baseline, with 95\% confidence intervals (CIs) and nominal $\mathrm{p}$ values, using paired t-tests. Stratification was also carried out concurrently by injection frequency and baseline VA.

\section{RESULTS}

\section{Patient characteristics}

Based on eligibility criteria, this study evaluated 15613 patient eyes in the 6-month cohort and 12214 patient eyes in the 1-year cohort; these cohorts were not mutually exclusive, but the 1-year cohort was smaller due to the requirement for a longer 1-year follow up within the study inclusion dates, as well as patient dropout. Table 1 summarises baseline demographics. In the 6-month cohort, in those 8876 patient eyes with BRVO-related $\mathrm{ME}$, the mean age at presentation was 71.8 years and $55 \%$ were women. Mean baseline VA was 55.1 letters (20/80 Snellen equivalent). Initial anti-VEGF agents were distributed as follows: $14 \%$ aflibercept (1271 eyes), 36\% ranibizumab (3214 eyes) and $49 \%$ bevacizumab (4391 eyes). The baseline characteristics of the 1-year BRVO-related ME cohort were very similar.

In those 6737 patient eyes with CRVO-related ME within the 6 -month cohort, the mean age at presentation was 72.8 years and $51 \%$ were women. Mean baseline VA was 37.2 letters (20/200 Snellen equivalent). Initial anti-VEGF agents were distributed as follows: $20 \%$ aflibercept (1374 eyes), 32\% ranibizumab (2140 eyes) and $48 \%$ bevacizumab (3223 eyes). The baseline characteristics of the 1-year CRVO-related ME cohort were very similar. In addition, while all patients in this analysis received anti-VEGF therapy, a minority of patient eyes also underwent focal laser and panretinal laser or intravitreal corticosteroids, as detailed in table 1, reflecting the real-world nature of this study. Of note, the mean number of anti-VEGF injections was over 60 times greater and over 40- imes greater than the mean number for any of these treatments over 6 months and 1 year, respectively.

\section{Mean change in visual acuity}

Table 1 also summarises mean VA change in 6 months and 1 year. In 6 months, for BRVO-related ME, after a mean of 4.5 antiVEGF injections, there was a mean gain of 9.4 letters (95\% CI for change in VA, +8.94 to $+9.78 ; \mathrm{p}<0.001)$. Similarly, in 1 year, after a mean of 7.4 anti-VEGF injections, there was a mean gain of 8.1 letters $(95 \%$ CI for change in VA, +7.55 to $+8.57 ; \mathrm{p}<0.001)$. For CRVO-related ME, in 6 months, after a mean of 4.6 antiVEGF injections, there was a mean gain of 9.2 letters (95\% CI for change in VA, +8.50 to $+9.87 ; \mathrm{p}<0.001)$. Likewise, in 1 year, after a mean of 7.6 anti-VEGF injections, there was a mean gain of 7.1 letters $(95 \%$ CI for change in VA, +6.31 to $+7.95 ; \mathrm{p}<0.001)$.

Assessment, excluding patients with RVO who received bilateral treatment during the 6-year study period, yielded nearly identical outcomes, as only $3 \%$ of patients with BRVO and $4 \%$ of patients with CRVO were treated bilaterally during this time.

The analysis was also stratified by anti-VEGF agents, excluding patient eyes that switched between anti-VEGF agents. In the 6-month cohort, in those eyes with BRVO-related ME, baseline VAs were well balanced and the outcomes were clinically similar. Specifically, 1157 patient eyes were treated with a mean of 4.2 aflibercept-only injections and improved by 9.6 letters, while 3255 were treated with a mean of 4.3 bevacizumab-only injections, improving by 10.1 letters, and 2876 were treated with a mean of 4.5 ranibizumab-only injections, improving by 8.8 letters. In 1 year, outcomes stratified by anti-VEGF agents were similar (mean 6.9 aflibercept-only injections in 845 patient eyes yielding 7.8 letters, mean 6.6 bevacizumab-only injections in 2106 patient eyes yielding 8.2 letters and mean of 4.5 ranibizumab-only injections in 2081 patient eyes yielding 7.9 letters).

In those eyes with CRVO-related ME, baseline VAs were not balanced when stratified by anti-VEGF agents, with the lowest baseline VA group improving most, possibly reflecting a ceiling effect and potentially confounding outcomes. In the 6-month cohort, baseline VAs were 36.8, 35.8 and 39.7 letters for 1268 aflibercept-only injected eyes, 2364 bevacizumab-only injected eyes and 1879 ranibizumab-only injected eyes, with improvements of 8.8, 11.2 and 7.4 letters, despite similar injections of 4.3, 4.3 and 4.6, respectively. In the 1-year cohort, baseline VAs were $38.8,39.4$ and 40.3 in 910 aflibercept-only injected eyes, 1569 bevacizumab-only injected eyes and 1400 ranibizumabonly eyes with improvements of 7.3, 8.4 and 5.7 letters after 7.0, 6.8 and 7.7 injections, respectively.

\section{Anti-VEGF treatment intensity and baseline features}

Figure 1, a series of histograms, depicts the distribution of patient eyes stratified by number of injections in 6 months between 1 and 7, and in 1 year, between 1 and 13. In BRVO-related ME, patient eyes received a mean of 4.5 with a median of 5 anti-VEGF injections in 6 months and a mean of 7.4 with a median of 8 injections in 1 year. In CRVO-related ME, patient eyes received a mean of 4.6 with a median of 5 anti-VEGF injections in 6 months and a mean of 7.6 anti-VEGF injections with a median of 8 injections in 1 year. The distribution of injections/eye is not normal and shows negative skew. For example, in both BRVO-related and CRVO-related ME, nearly $25 \%$ of patient eyes received three or 
Table 1 Baseline features and outcomes

\begin{tabular}{|c|c|c|c|c|}
\hline \multicolumn{5}{|c|}{ Stratified by diagnosis and follow-up interval } \\
\hline & $\begin{array}{l}\text { BRVO } \\
6 \text { months }\end{array}$ & $\begin{array}{l}\text { BRVO } \\
1 \text { year }\end{array}$ & $\begin{array}{l}\text { CRVO } \\
6 \text { months }\end{array}$ & $\begin{array}{l}\text { CRVO } \\
1 \text { year }\end{array}$ \\
\hline No. of patient eyes & 8876 & 6914 & 6737 & 5300 \\
\hline Female sex & $55 \%$ & $56 \%$ & $51 \%$ & $51 \%$ \\
\hline Mean baseline age (years) & 71.8 & 72.3 & 72.8 & 72.9 \\
\hline \multicolumn{5}{|l|}{ Mean baseline VA } \\
\hline Letters & 55.1 & 56.6 & 37.2 & 39.5 \\
\hline Snellen equivalent & $20 / 80$ & $20 / 80$ & $20 / 200$ & $20 / 160$ \\
\hline \multicolumn{5}{|l|}{ Initial anti-VEGF agent } \\
\hline Bevacizumab & $49 \%$ & $49 \%$ & $48 \%$ & $46 \%$ \\
\hline Aflibercept & $14 \%$ & $14 \%$ & $20 \%$ & $21 \%$ \\
\hline Ranibizumab & $36 \%$ & $37 \%$ & $32 \%$ & $33 \%$ \\
\hline Mean no. of anti-VEGF injections & 4.5 & 7.4 & 4.6 & 7.6 \\
\hline Median no. of anti-VEGF injections & 5 & 8 & 5 & 8 \\
\hline \multicolumn{5}{|l|}{ Other treatments } \\
\hline Steroid injections, mean \# & 0.06 & 0.15 & 0.06 & 0.17 \\
\hline Steroid injections, \% patients & $5 \%$ & $9 \%$ & $8 \%$ & $9 \%$ \\
\hline Focal laser, mean \# & 0.07 & 0.13 & 0.01 & 0.03 \\
\hline Focal laser, $\%$ patients & $7 \%$ & $12 \%$ & $1 \%$ & $2 \%$ \\
\hline Panretinal laser, mean \# & 0.02 & 0.03 & 0.04 & 0.09 \\
\hline Panretinal laser, $\%$ patients & $2 \%$ & $3 \%$ & $5 \%$ & $6 \%$ \\
\hline Mean VA change ( letters) & 9.4 & 8.1 & 9.2 & 7.1 \\
\hline$P$ value for change & $<0.001$ & $<0.001$ & $<0.001$ & $<0.001$ \\
\hline $\mathrm{Cl}(95 \%)$ & 8.94 to 9.78 & 7.55 to 8.57 & 8.50 to 9.87 & 6.31 to 7.95 \\
\hline \multicolumn{5}{|c|}{ Stratified by diagnosis, follow-up interval and baseline visual acuity } \\
\hline & $20 / 40$ or better & 20/40-20/70 & $20 / 70-20 / 200$ & $20 / 200$ or worse \\
\hline \multicolumn{5}{|l|}{ BRVO 6 months } \\
\hline No. of patient eyes & 2428 & 2946 & 2442 & 1060 \\
\hline$\%$ Patient eyes & $27 \%$ & $33 \%$ & $28 \%$ & $12 \%$ \\
\hline Female sex & $53 \%$ & $55 \%$ & $55 \%$ & $59 \%$ \\
\hline Mean baseline age (years) & 69.3 & 72.2 & 72.9 & 73.9 \\
\hline Mean no. of anti-VEGF injections & 4.3 & 4.5 & 4.6 & 4.8 \\
\hline Mean VA change (letters) & -0.2 & 5.2 & 12.9 & 34.6 \\
\hline$P$ value for change & 0.318 & $<0.001$ & $<0.001$ & $<0.001$ \\
\hline $\mathrm{Cl}(95 \%)$ & -0.53 to 0.17 & 4.77 to 5.64 & 12.22 to 13.61 & 32.51 to 36.69 \\
\hline \multicolumn{5}{|l|}{ BRVO 1 year } \\
\hline No. of patient eyes & 2041 & 2363 & 1786 & 715 \\
\hline$\%$ Patient eyes & $30 \%$ & $34 \%$ & $26 \%$ & $10 \%$ \\
\hline Female sex & $53 \%$ & $55 \%$ & $58 \%$ & $61 \%$ \\
\hline Mean baseline age (years) & 70.0 & 72.7 & 73.8 & 73.7 \\
\hline Mean no. of anti-VEGF injections & 7.0 & 7.4 & 7.5 & 7.5 \\
\hline Mean VA change (letters) & -1.3 & 4.2 & 12.0 & 37.6 \\
\hline $\mathrm{P}$ value for change & $<0.001$ & $<0.001$ & $<0.001$ & $<0.001$ \\
\hline $\mathrm{Cl}(95 \%)$ & -1.76 to -0.81 & 3.64 to 4.79 & 11.02 to 12.89 & 35.02 to 40.21 \\
\hline \multicolumn{5}{|l|}{ CRVO 6 months } \\
\hline No. of patient eyes & 954 & 1620 & 1995 & 2167 \\
\hline$\%$ Patient eyes & $14 \%$ & $24 \%$ & $30 \%$ & $32 \%$ \\
\hline Female sex & $47 \%$ & $50 \%$ & $49 \%$ & $53 \%$ \\
\hline Mean baseline age (years) & 68.7 & 71.9 & 73.0 & 74.9 \\
\hline Mean no. of anti-VEGF injections & 4.3 & 4.5 & 4.6 & 4.7 \\
\hline Mean VA change (letters) & -4.5 & 0.3 & 7.0 & 23.9 \\
\hline$P$ value for change & $<0.001$ & 0.529 & $<0.001$ & $<0.001$ \\
\hline $\mathrm{Cl}(95 \%)$ & -5.6 to -3.45 & -0.68 to 1.28 & 5.91 to 8.04 & 22.41 to 25.37 \\
\hline
\end{tabular}




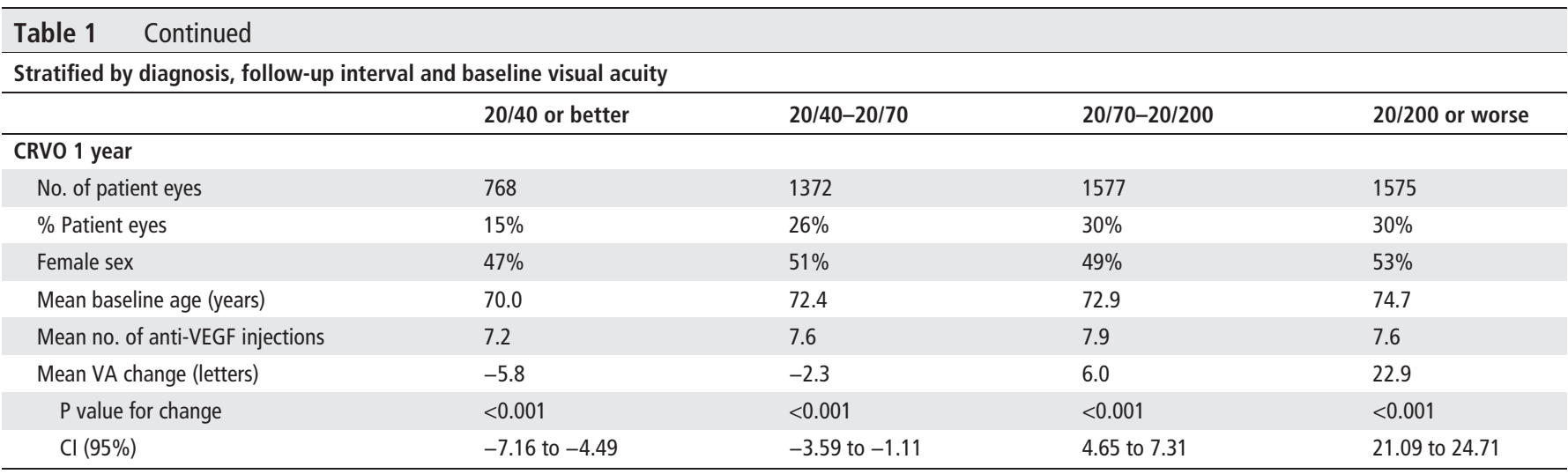

BRVO, branch retinal vein occlusion; CRVO, central retinal vein occlusion; VA, visual acuity; VEGF, vascular endothelial growth factor.

BRVO

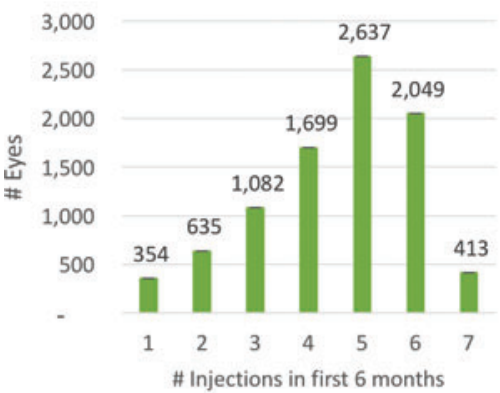

CRVO
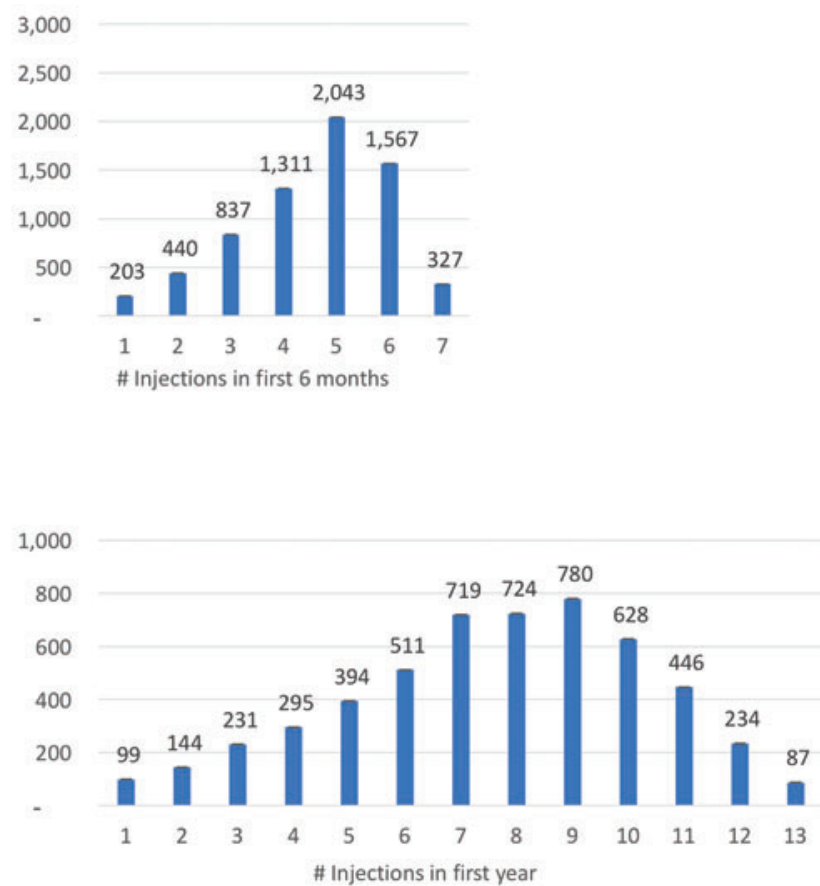

Figure 1 Histograms showing distribution of patient eyes with retinal vein occlusion stratified by number of antivascular endothelial growth factor injections received in 6 months and 1 year. BRVO, branch retinal vein occlusion; CRVO, central retinal vein occlusion.

fewer injections in 6 months and nearly 33\% of patient eyes received six or fewer injections in 1 year.

Table 2 summarises baseline features stratified by the number of anti-VEGF injections over 6 months and 1 year. For both BRVO-related and CRVO-related ME, the mean number of antiVEGF injections over 6 months and over 1 year did not trend with baseline age or baseline VA, except in those patients receiving six or seven injections in 6 months who tended to show baseline VA worse than the overall mean.

\section{Visual outcomes stratified by anti-VEGF treatment intensity and baseline visual acuity}

For both BRVO-related and CRVO-related ME, when stratified by anti-VEGF injection frequency, final VA in 6 months and 1 year generally increased with increasing treatment intensity, as summarised in table 2 and figure 2. Also, mean 1 -year VA change broadly demonstrated a linear relationship with mean number of anti-VEGF injections, especially beyond two injections, as depicted in table 2 and figure 2 . At the lower range, those patient eyes that received $\leq 3$ injections in 6 months or $\leq 6$ injections in 1 year improved minimally, generally gaining $\leq 5$ letters in 6 months or 1 year.

When stratified by baseline VA, visual outcomes demonstrated ceiling effects, as summarised in table 1 and figure 3 . In particular, stratified by baseline VA of 20/200 or worse, 20/70 to 20/200, 20/ 40 to $20 / 70$ and $20 / 40$ or better, mean number of injections was similar, but there was a consistent trend of diminishing improvement with better baseline VA in both BRVO-related and CRVOrelated ME in 6 months and 1 year. In addition, despite greater improvement with worse baseline VA, the final 6-month and 1-year VA remained in the same stratum as the baseline VA. For instance, although patient eyes with baseline VA of 20/40 or better generally risked VA mild loss in 1 year, they generally demonstrated better VA in both 6 months and 1 year versus those with worse baseline VA. 
Table 2 Mean 6-month and 1-year changes in visual acuity stratified by diagnosis, follow-up interval and anti-vascular endothelial growth factor injection frequency

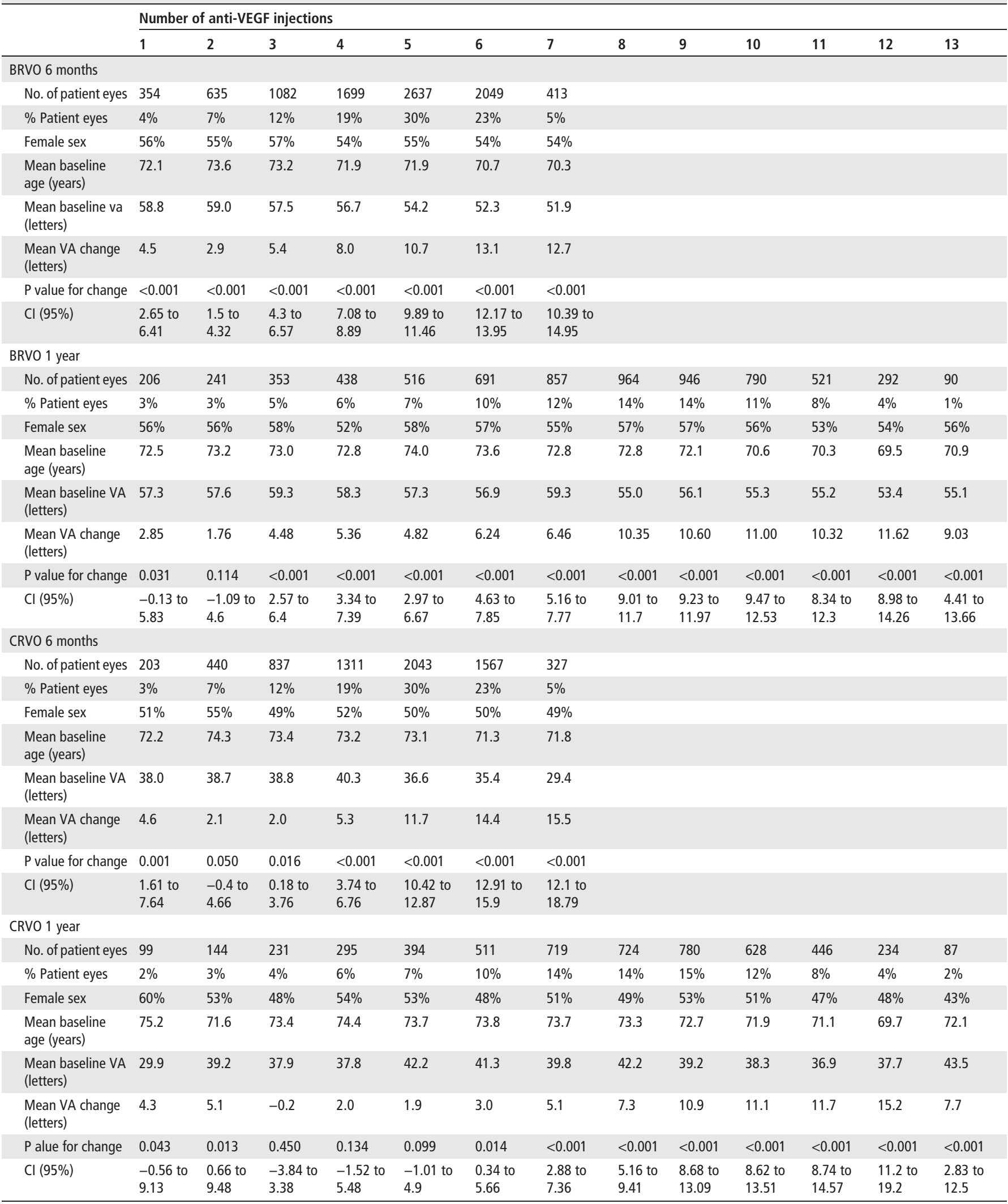

BRVO, branch retinal vein occlusion; CRVO, central retinal vein occlusion; VA, visual acuity; VEGF, vascular endothelial growth factor.

For both BRVO-related and CRVO-related ME in 6 months and 1 year, when stratified by both anti-VEGF injection frequency and baseline VA, subgroup size became limiting. However, compared with BRVO-related ME, patient eyes with CRVO-related ME generally performed worse for a given injection frequency and baseline VA. In both BRVO-related and CRVO-related ME, those patient eyes with baseline VA of 20/40 or better risked mild VA loss in both 6 months and 1 year, regardless of injection frequency. In 

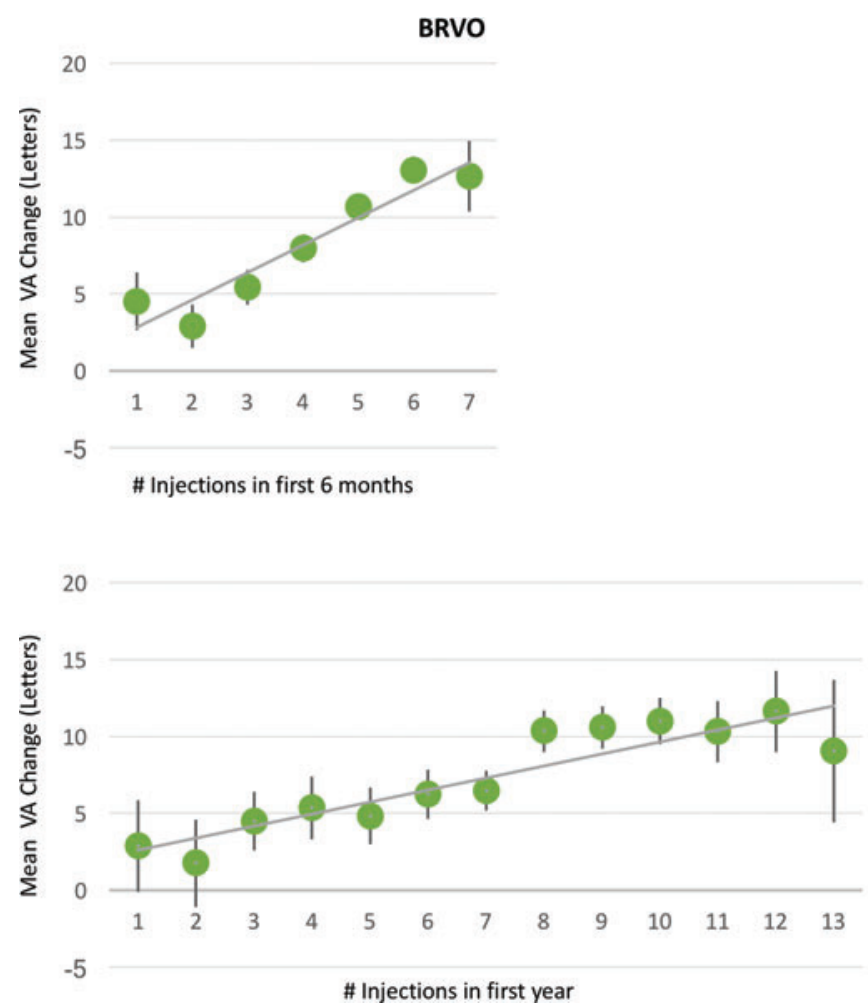

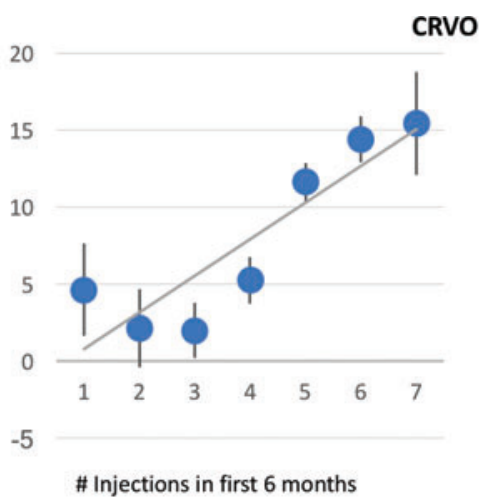

\# Injections in first 6 months

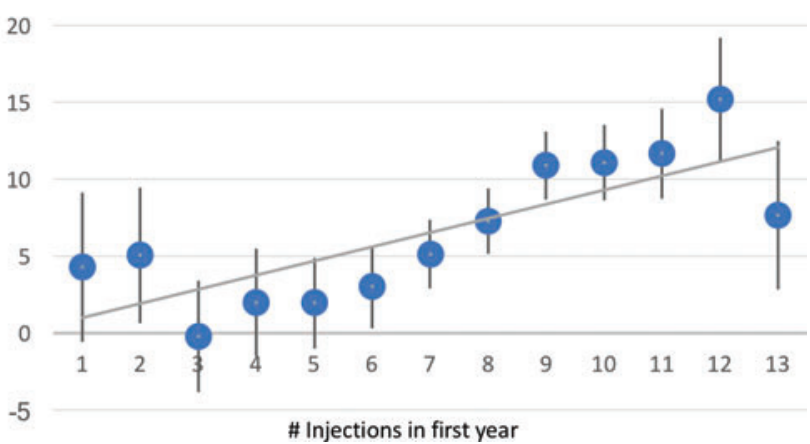

Figure 2 Graphs showing change in visual acuity (VA) versus antivascular endothelial growth factor injections administered to all patient eyes with retinal vein occlusion in 6 months and 1 year. The $95 \% \mathrm{Cls}$ are included. Mean VA change tended to increase with greater treatment intensity. BRVO, branch retinal vein occlusion; CRVO, central retinal vein occlusion.

contrast, those patient eyes with moderately severe VA loss at presentation (baseline VA between 20/70 and 20/200) who received $\geq 5$ injections in 6 months or $\geq 9$ injections in 1 year improved by approximately 15 and 10 letters for BRVO-related and CRVOrelated ME, respectively.

\section{DISCUSSION}

This real-world study is limited by its retrospective design, nonstandardised VA assessment and non-standardised diagnostic criteria for ME and RVO. These limitations may partly account for different outcomes in real-world studies compared with RCTs, which include protocol BCVA testing as well as explicit inclusion and exclusion criteria. However, the strengths of this study include the large sample size which facilitated stratification to further evaluate injection frequency and baseline VA. Notably, this study yields relevant insights on unmet need, as the healthcare system adopts value-based approaches with increasing accountability for clinical practice outcomes. In particular, this US-based study demonstrates that patients receive fewer antiVEGF injections and experience less visual improvement by approximately 2 lines of vision (10 letters), compared with patients undergoing protocol-based anti-VEGF therapy in randomised controlled trials (RCTs). In addition, visual outcomes generally correlate with treatment intensity in 6 months and in 1 year, with ceiling effects related to baseline VA.

\section{Real-world patients with RVO-related ME receive fewer anti-VEGF injections and experience worse outcomes compared with RCTs}

In clinical practice, patients with RVO-related ME undergo fewer anti-VEGF injections and experience worse visual outcomes, compared with patients receiving therapy in RCTs. This conclusion suggests unmet needs related to treatment burden with current medications. In particular, for BRVO-related ME, VA improved by 9.4 letters after a mean of 4.5 anti-VEGF injections in 6 months and improved by 8.1 letters after a mean of 7.4 anti-VEGF injections in 1 year. These results are consistent with the one large real-world analysis of outcomes, in BRVO-related ME, from the UK National Health Service EMR system, in which 1598 patient eyes treated with a mean of 3.8 anti-VEGF injections gained 8.8 letters in 6 months; 1042 patient eyes treated with a mean of 5.1 anti-VEGF injections gained 9.6 letters in 1 year. $^{7}$

In contrast, theBRAVO registration trial for ranibizumab demonstrated an improvement of 18.3 letters in both 6 months and 1 year. ${ }^{8}$ In BRAVO, patients underwent monthly treatment for the first 6 months, followed by as-needed treatment for the second 6 months (mean 2.7 injections). ${ }^{9}$ Of note, while $7 \%$ of the real-world patient eyes underwent focal laser treatment over 6 months, an even larger $19.8 \%$ of ranibizumab-treated patient eyes in BRAVO also underwent focal laser over the same time period. ${ }^{8}$ For aflibercept, the VIBRANT registration trial showed a similar improvement to BRAVO, with 17.0 and 17.1 letter gain in 6 and 12 months, respectively, using monthly treatment for the first 6 months, followed by bimonthly treatment for the second 6 months. ${ }^{10} 11$

For CRVO-related ME, in the current study, VA improved by 9.2 letters after a mean of 4.6 anti-VEGF injections over 6 months and improved by 7.1 letters after a mean of 7.6 anti-VEGF injections in 1 year. In contrast, the CRUISE registration trial for ranibizumab showed a BVCA improvement of 14.9 letters and 13.9 letters in 6 months and 1 year, respectively. ${ }^{12}{ }^{13}$ In CRUISE, patients underwent monthly treatment for the first 6 months, followed by as-needed treatment for the second 6 months (mean 3.3 injections). In two registration trials for aflibercept (COPERNICUS and GALILEO, respectively), BVCA improved 

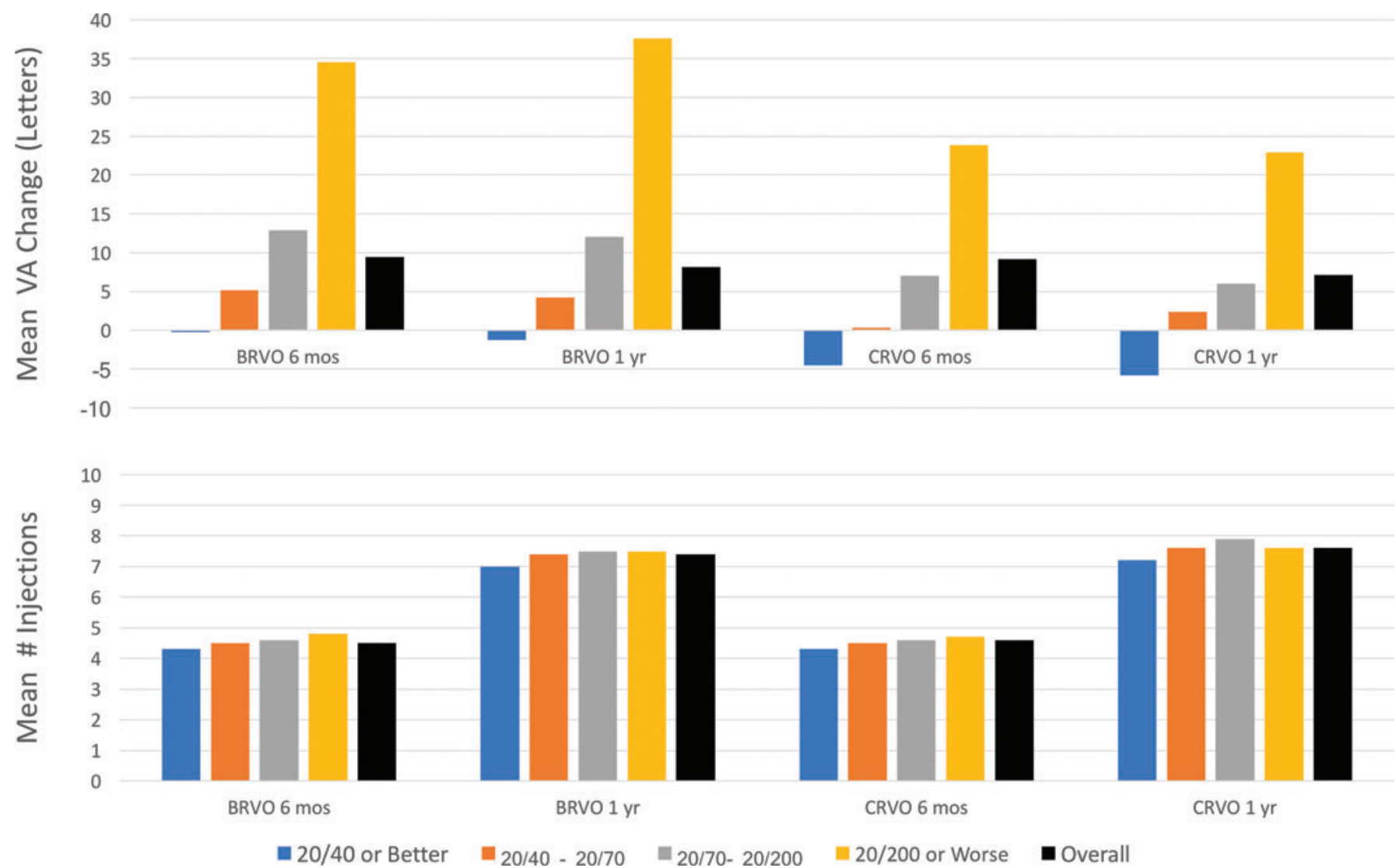

Figure 3 Graphs showing the mean number of anti-vascular endothelial growth factor injections and mean visual acuity (VA) change in patient eyes with retinal vein occlusion over 6 months and 1 year, overall and stratified by baseline VA. There were ceiling effects related to baseline VA; mean VA change tended to increase in patient eyes with decreased baseline VA, despite similar treatment intensity. BRVO, branch retinal vein occlusion; CRVO, central retinal vein occlusion.

by 17.3 letters and 18.0 letters in 6 months and by 16.2 letters and 16.9 letters in 1 year. ${ }^{14} 15$ Patients underwent monthly treatment for the first 6 months, followed by as-needed treatment for the second 6 months (mean 2.7 and 2.5 injections, respectively). Similarly, the SCORE2 trial in CRVO-related ME showed a 19letter and 18.9-letter improvement in 6 months after monthly aflibercept and bevacizumab, respectively. ${ }^{16}$

Cross-trial comparisons are limited by differing eligibility criteria, therapeutic regimens and endpoint evaluations, including non-standardised VA assessments in real-world studies. For instance, the real-world patients in this study were slightly older than those patients in the previously discussed RCTs, with similar baseline VA for patients with BRVO, but with worse baseline VA for patients with CRVO; some patients also underwent additional therapies as summarised above, but some of the RCTs also included laser rescue. Also, real-world studies tend towards worse therapeutic outcomes, given the more diverse patient presentations, likely including advanced disease states such as ischaemia, which may be ineligible for RCTs. Nonetheless, while the precise differences in visual gains compared with RCTs may be uncertain, this current study strongly supports a meaningful shortfall, highlighting the unmet need for more efficacious and longer-acting agents to address treatment burden.

In the current real-world study, the comparatively lower treatment intensity suggests that physicians use as-needed or treat-andextend dosing regimens that strive to decrease treatment burden. The distribution of injections/eye, as depicted in figure 1, further supports this premise, as it is not normal and shows negative skew, with nearly $25 \%$ patient eyes undergoing three or fewer injections in 6 months and nearly 33\% patient eyes undergoing 6 or fewer injections in 1 year. Notably, age or baseline VA did not affect treatment frequency decisions, as those who received the fewest injections in 6 months or 1 year generally did not differ meaningfully from the overall group. Therefore, undertreatment may partially account for worse visual outcomes in real-world patients with RVO, in addition to more diverse patient presentations and advanced disease states typically ineligible for RCTs.

Visual outcomes correlate with treatment intensity in 1 year, with ceiling effects related to baseline VA

While patients with RVO in the previously discussed RCTs underwent six fixed monthly treatments initially, nearly $25 \%$ of patient eyes in the current study received three or fewer injections in 6 months. When stratified by anti-VEGF injection frequency, 1-year VA gain increased with increasing treatment intensity on average, which is not unexpected. For both BRVO-related and CRVO-related ME, there was generally a linear relationship between mean letters gained and mean number of anti-VEGF injections in 1 year, with a gain of nearly 1 letter for each addition anti-VEGF injection. At the lower range, those patient eyes that received $\leq 5$ injections in 1 year typically improved minimally, generally gaining less than 1 line of vision. Those who underwent $\geq 9$ injections in 1 year generally gained more than 2 lines of vision. Given the $\leq 9$-day half-life of current anti-VEGF agents, ${ }^{17-21}$ these results are not surprising, as fewer treatments would only intermittently inhibit VEGF. As noted previously, these results highlight the need for longer-acting therapies to address treatment burden associated with current anti-VEGF agents. 
When stratified by baseline VA, results were consistent with the aforementioned real-world outcomes study in BRVO-related ME, from the UK National Health Service EMR system, in which the absolute improvement in VA from baseline was sequentially greater in each subsequently worse baseline VA quintile. ${ }^{7}$ In this UK-based study and in the current study, despite greater improvement with worse baseline VA, the 6-month and 1-year VA remained in the same stratum as the baseline VA; in other words, those patient eyes starting treatment with lower VA also remained with lower VA and vice versa. In addition, in the current study, for both BRVO-related and CRVO-related ME, patients with good baseline VA, 20/40 or better, generally lost a small number of letters, reflecting a ceiling effect, which can limit improvement in eyes with relatively good baseline VA.

When stratified by both baseline VA and treatment intensity, subgroup size became limiting. In general, however, the mean VA change improved with both increased anti-VEGF injection frequency and decreased baseline VA. However, compared with BRVO-related ME, patient eyes with CRVOrelated ME gnerally performed worse for a given injection frequency and baseline VA. This result is not unexpected as CRVO generally more severely compromises macular perfusion compared to BRVO. In both BRVO-related and CRVOrelated ME, those patient eyes with baseline VA of 20/40 or better risked mild VA loss at both 6 months and 1 year, regardless of injection frequency. In contrast, those patient eyes with moderately severe VA loss at presentation (baseline VA between 20/70 and 20/200) improved by approximately 15 and 10 letters for BRVO-related and CRVO-related ME, respectively, when they received $\geq 5$ injections in 6 months or $\geq 9$ injections in 1 year, a treatment intensity somewhat similar to registration trials. This outcome reassuring demonstrates that more intense therapy with current anti-VEGF agents yields meaningful benefit in clinical practice.

\section{Patients with RVO experience greater 1-year gain than patients with AMD and DME, but exhibit a larger gap in visual gain when compared with corresponding randomised controlled trials}

As noted previously, cross-trial comparisons are limited, but can provide clinical context across common retinal disorders treated with current anti-VEGF agents. Importantly, the realworld 1-year VA gain for RVO-related ME favourably compares with the real-world 1-year VA gain for nAMD and DME, assessed with the same database using similar analysis methodology. In particular, real-world patients with BRVOrelated and CRVO-related ME gain 8.1 and 7.1 letters after a mean of 7.4 and 7.6 injections, respectively, in 1 year, while a recent real-world study with corresponding eligibility criteria in 49485 patient eyes with nAMD showed only a 1.0 letter gain after a mean of 7.3 anti-VEGF injections. ${ }^{4}$ Similarly, a recent real-world study with corresponding eligibility criteria in 28658 patient eyes with DME demonstrated a mean gain of 4.2 letters after a mean of 6.4 anti-VEGF injections in 1 year. $^{5}$

Nonetheless, while real-world patients with RVO-related ME show greater 1-year visual gains than real-world patients with nAMD and DME, they experience a larger gap in visual gain when compared with corresponding RCTs. In particular, for BRVO-related and CRVO-related ME, the aforementioned registration trials demonstrate an average 1-year BCVA improvement of 17.7 letters and 15.7 letters, respectively. ${ }^{9} 101214 \quad 15$ For nAMD, registration trials for aflibercept and ranibizumab, as well as the monthly bevacizumab and ranibizumab arms of the Comparison of Age-Related Macular Degeneration Treatments
Trials, suggest that these anti-VEGF agents perform similarly, providing an average 1-year BCVA improvement of 8.7 letters. ${ }^{22-25}$ Similarly, for DME, registration trials for aflibercept and ranibizumab, as well as the Diabetic Retinopathy Clinical Research Network comparative effectiveness trial (Protocol T), show an average 1-year BCVA improvement of 10.9 letters with these anti-VEGF agents. ${ }^{26-28}$

\section{CONCLUSION}

In summary, patients with RVO-related ME in the US experience meaningfully worse 6-month and 1-year VA gains compared with patients in RCTs. However, the mean VA change generally improved in patient eyes with both increased anti-VEGF injection frequency and decreased baseline VA, with a ceiling effect associated with baseline VA. Specifically, real-world patient eyes with RVO-related ME with relatively good baseline VA generally risk VA loss in 1 year, regardless of treatment intensity. In contrast, those with moderately severe VA loss at presentation who received treatment intensity somewhat similar to registration trials generally improved by approximately 3 lines and 2 lines for BRVO-reated and CRVO-related ME, respectively, which reassuringly demonstrates the potential of current anti-VEGF medications. Real-world 1-year outcomes for RVO-related ME favourably compare with those for nAMD and DME, assessed with the same database and similar analysis methodology, but real-world patients with RVO-related ME experience a larger gap in visual gain when compared with corresponding RCTs. Importantly, as the healthcare system adopts value-based approaches, these clinical outcome studies assume greater importance, particularly for benchmarking. Finally, these results highlight the need for appropriate patient counselling regarding treatment frequency with current anti-VEGF agents and also highlight the unmet need for more effective and longer-acting therapies, in order to address treatment burden.

\section{Twitter Thomas Ciulla @ThomasCiullaMD.}

Contributors Each author has made substantial contributions to the conception and design of the work, analysis and interpretation of data. Each author agrees to be accountable for all aspects of the work with respect to its accuracy and integrity.

Funding The authors have not declared a specific grant for this research from any funding agency in the public, commercial or not-for-profit sectors.

Competing interests Drs Pollack and Williams are co-founders of Vestrum Health, whose database was utilised for this study.

Provenance and peer review Not commissioned; externally peer reviewed. Data availability statement Data are available upon reasonable request.

Open access This is an open access article distributed in accordance with the Creative Commons Attribution Non Commercial (CC BY-NC 4.0) license, which permits others to distribute, remix, adapt, build upon this work non-commercially, and license their derivative works on different terms, provided the original work is properly cited, appropriate credit is given, any changes made indicated, and the use is noncommercial. See: http://creativecommons.org/licenses/by-nc/4.0/.

\section{ORCID iD}

Thomas Ciulla http://orcid.org/0000-0001-5557-6777

\section{REFERENCES}

1 ASRS 2017. Preferences and trends membership survey. Chicago, IL: American Society of Retina Specialists, 2017.

2 Ciulla TA, Bracha P, Pollack J, et al. Real-world outcomes of anti-vascular endothelial growth factor therapy in diabetic macular edema in the United States. Ophthalmol Retina 2018;2:1179-1187.

3 Ciulla TA, Huang F, Westby K, et al. Real-world outcomes of anti-vascular endothelial growth factor therapy in neovascular age-related macular degeneration in the United States. Ophthalmol Retina 2018;2:645-653.

4 Ciulla TA, Hussain RM, Pollack JS, et al. Visual acuity outcomes and anti-vascular endothelial growth factor therapy intensity in neovascular age-related macular 
degeneration patients: a real-world analysis of 49485 eyes. Ophthalmol Retina 2020:4:19-30.

5 Ciulla TA, Pollack JS, Williams DF. Visual acuity outcomes and anti-VEGF therapy intensity in diabetic macular oedema: a real-world analysis of 28658 patient eyes. $\mathrm{Br}$ J Ophthalmol 2020. Published Online First.

6 Gregori NZ, Feuer W, Rosenfeld PJ. Novel method for analyzing snellen visual acuity measurements. Retina 2010;30:1046-50.

7 Gale R, Pikoula M, Lee AY, et al. Real world evidence on 5661 patients treated for macular oedema secondary to branch retinal vein occlusion with intravitreal anti-vascular endothelial growth factor, intravitreal dexamethasone or macular laser. Br J Ophthalmol 2020

8 Campochiaro PA, Heier JS, Feiner L, et al. Ranibizumab for macular edema following branch retinal vein occlusion: six-month primary end point results of a phase III study. Ophthalmology 2010;117:1102-1112 e1.

9 Brown DM, Campochiaro PA, Bhisitkul RB, et al. Sustained benefits from ranibizumab for macular edema following branch retinal vein occlusion: 12-month outcomes of a phase III study. Ophthalmology 2011;118:p.1594-602.

10 Clark WL, Boyer DS, Heier JS, et al. Intravitreal aflibercept for macular edema following branch retinal vein occlusion: 52-week results of the vibrant study. Ophthalmology 2016;123:330-336.

11 Campochiaro PA, Clark WL, Boyer DS, et al. Intravitreal aflibercept for macular edema following branch retinal vein occlusion: the 24-week results of the VIBRANT study. Ophthalmology 2015;122:538-44

12 Campochiaro PA, Brown DM, Awh CC, et al. Sustained benefits from ranibizumab for macular edema following central retinal vein occlusion: twelve-month outcomes of a phase III study. Ophthalmology 2011;118:p.2041-9.

13 Brown DM, Campochiaro PA, Singh RP, et al. Ranibizumab for macular edema following central retinal vein occlusion: six-month primary end point results of a phase III study. Ophthalmology 2010;117:1124-1133 e1.

14 Brown DM, Heier JS, Clark WL, et al. Intravitreal aflibercept injection for macular edema secondary to central retinal vein occlusion: 1-year results from the phase 3 COPERNICUS study. Am J Ophthalmol 2013;155:429-437 e7.
15 Korobelnik JF, Holz FG, Roider J, et al. Intravitreal aflibercept injection for macular edema resulting from central retinal vein occlusion: one-year results of the phase 3 galileo study. Ophthalmology 2014;121:p.202-208.

16 Scott IU, Vanveldhuisen PC, Ip MS, et al. Effect of bevacizumab vs aflibercept on visual acuity among patients with macular edema due to central retinal vein occlusion: the SCORE2 randomized clinical trial. JAMA 2017;317:p.2072-2087.

17 Fogli S, Del Re M, Rofi E, et al. Clinical pharmacology of intravitreal anti-VEGF drugs. Eye (Lond) 2018;32:1010-1020.

18 Krohne TU, Liu Z, Holz FG, et al. Intraocular pharmacokinetics of ranibizumab following a single intravitreal injection in humans. Am J Ophthalmol 2012;154:682-686 e2.

19 Stewart MW. What are the half-lives of ranibizumab and aflibercept (VEGF Trap-eye) in human eyes? Calculations with a mathematical model. Eye Rep 2011;1:12-14.

20 Stewart MW, Rosenfeld PJ, Penha FM, et al. Pharmacokinetic rationale for dosing every 2 weeks versus 4 weeks with intravitreal ranibizumab, bevacizumab, and aflibercept (vascular endothelial growth factor Trap-eye). Retina 2012;32:434-57.

21 Stewart MW. Pharmacokinetics, pharmacodynamics and pre-clinical characteristics of ophthalmic drugs that bind VEGF. Expert Rev Clin Pharmacol 2014;7:167-80.

22 Brown DM, Kaiser PK, Michels M, et al. Ranibizumab versus verteporfin for neovascular age-related macular degeneration. N Engl J Med 2006;355:1432-44.

23 Martin DF, Maguire MG, Ying GS, et al. Ranibizumab and bevacizumab for neovascular age-related macular degeneration. N Engl J Med 2011;364:1897-908.

24 Heier JS, Brown DM, Chong V, et al. Intravitreal aflibercept (VEGF trap-eye) in wet age-related macular degeneration. Ophthalmology 2012;119:2537-48.

25 Rosenfeld PJ, Brown DM, Heier JS, et al. Ranibizumab for neovascular age-related macular degeneration. N Engl J Med 2006;355:1419-31.

26 Korobelnik JF, Do DV, Schmidt-Erfurth U, et al. Intravitreal aflibercept for diabetic macular edema. Ophthalmology 2014;121:2247-54.

27 Nguyen QD, Brown DM, Marcus DM, et al. Ranibizumab for diabetic macular edema: results from 2 phase III randomized trials: RISE and RIDE. Ophthalmology 2012;119:789-801.

28 Wells JA, Glassman AR, Ayala AR, et al. Aflibercept, bevacizumab, or ranibizumab for diabetic macular edema. N Engl J Med 2015;372:1193-203. 